\title{
Semioccluded Vocal Tract Exercises Improve Self-Perceived Voice Quality in Healthy Actors
}

\author{
*Valentina Di Natale, ${ }^{*}{ }^{\dagger}$ Giovanna Cantarella, ${ }^{\ddagger}$ Claudia Manfredi, ${ }^{*}$ Annaclara Ciabatta, ${ }^{\ddagger}$ Cosimo Bacherini, and \\ ${ }^{\S}$ Philippe H. DeJonckere, ${ }^{*} \dagger$ Milan, and $\ddagger$ Firenze, Italy, and $\S$ Brussels, Belgium
}

\begin{abstract}
Summary: Purpose. Semi-occluded vocal tract exercises (SOVTE) have shown to lead to more effective and efficient vocal production for individuals with voice disorders and for singers. The aim of the present study is to investigate the effects of a 10-minute SOVTE warm-up protocol on the actors' voice.

Methods. Twenty-seven professional theater actors (16 females) without voice complaints were audio-recorded while reading aloud, with their acting voice, a short dramatic passage at four time points. Recordings were made: the day before the show, just before and soon after the warm-up protocol which was performed prior to the show and soon after the show. The voice quality was acoustically and auditory-perceptually evaluated and quantified at each time point by blinded raters. Self-assessment parameters anonymously collected pre and post exercising were also analyzed.
\end{abstract}

Results. No statistically significant differences on perceptual ratings and acoustic parameters were found between pre/post exercise sessions and males/females. A statistically significant improvement was detected in the self-assessment parameters concerning comfort of production, sonorousness, vocal clarity and power.

Conclusions. Vocal warm-up with the described SOVTE protocol was effective in determining a self-perceived improvement in comfort of production, voice quality and power, although objective evidence was missing. This straightforward protocol could thus be beneficial if routinely utilized by professional actors to facilitate the voice performance.

Key Words: Theater actors-Vocal warm-up-SOVTE-Voice analysis-Voice self-assessment—Actor's formant.

\section{INTRODUCTION}

Theater actors are voice professionals with high vocal demands. They need to project their voices to the audience in wide spaces, sometimes over the stage noise and often while engaging in emotionally loaded behaviors to interpret their character. ${ }^{1}$ Moreover, their vocal load can be increased by inappropriate stage conditions and improper or missing amplification. This leads to the necessity of voice production at high intensity levels with concomitant changes of the habitual pitch, ${ }^{2}$ and thus to potential laryngeal hyperfunction and consequent perceived vocal fatigue. ${ }^{3,4}$ In a study of Rangarathnam et al, ${ }^{5}$ auditory-perceptual and aerodynamic measures of theater actors' voices significantly deteriorated after 6 weeks of stage performances and rehearsals. Among actors, voice problems have shown to lead to both poor emotional health and occupational issues. ${ }^{3,6}$

The literature focusing on the effects of vocal warmup on healthy voices is various in terms of exercises which were investigated and outcomes which were considered. ${ }^{7}$ Exercises included in previous works can be

\footnotetext{
Presented at: Models and analysis of vocal emissions for biomedical applications, Firenze, Italy, 17-19 December 2019

Conflict of interest: None.

From the *Otolaryngology Department, Fondazione IRCCS Ca' Granda Policlinico Hospital, Milan, Italy; †Department of Clinical Sciences and Community Health, Milan University, Milan, Italy; †̦Department of Information Engineering, Università degli Studi di Firenze, Firenze, Italy; and the $§$ Federal Agency for Occupational Risks, Brussels, Belgium.

Address correspondence and reprint requests to Valentina Di Natale, Otolaryngology Department, Fondazione IRCCS Ca' Granda Ospedale Maggiore Policlinico, Milan, Italy. E-mail: dinatale.vale@tiscali.it

Journal of Voice, Vol. $\mathbf{\square}$, No. $\mathbf{\square}$, pp. $\mathbf{\square}-\mathbf{\square}$

0892-1997

(C) 2020 The Voice Foundation. Published by Elsevier Inc. All rights reserved.

https://doi.org/10.1016/j.jvoice.2020.07.024
}

grouped into four main categories: stretching, orofacial praxias, pitch variations and semi-occluded vocal tract exercises (SOVTE), these last being the most used. ${ }^{7}$ Positive effects of these exercises have been shown on acoustic, ${ }^{8-10}$ aerodynamic,,${ }^{8,11}$ auditory-perceptual ${ }^{11,12}$ and self-assessment ${ }^{13,14}$ parameters.

Previous studies on the effects of SOVTE showed that phonating while reducing the diameter of the vocal tract at the level of the tongue and the lips can result in a more effective and efficient vocal production on healthy and disordered voices and can improve singers' voices. ${ }^{15-18}$ According to Titze ${ }^{19}$ and Guzman et al, ${ }^{20}$ this effect is due to an increased oral pressure that favors a lower laryngeal position, a raised velum and a widened pharynx.

SOVTE can be differentiated according to the resistance created by the semi-occlusion, which directly relates to the amount of oral pressure which is generated. ${ }^{19,20}$ Amarante Andrade et $\mathrm{al}^{21}$ reported that SOVTE can also be classified according to the presence or absence of a vibratory component at the semi-occlusion level. In line with this model, exercises performed with a second source of vibration in addition to the vocal folds (named fluctuating) are characterized by a predominant massage effect on the vocal tract. Exercises performed with only the vibration of the vocal folds (named steady) promote easier phonation.

On healthy singers' voices, SOVTE have shown to improve self-assessment parameters such as voice emission comfort, harmonic quality, stability and cleanliness. ${ }^{22}$ Acoustically, a significant increase in intensity of the singer's formant and decrease in jitter and shimmer were detected. ${ }^{22,23}$

To the best of our knowledge, only one study ${ }^{24}$ has analyzed the effects of SOVTE on the actor's voice on a single 
subject detecting an increase of the speaker's formant as well as a better perceived voice quality after 30 minutes of SOVTE training.

The actor's/speaker's formant has been described as a strong energy peak around $3.5 \mathrm{kHz}$ in the long-term average spectrum (LTAS) of loud, trained, male speaking voices. ${ }^{25-27}$ It seems to be created by the clustering of the third, fourth and fifth formants (F3, F4, F5). As for the singer's formant, which consisted of a peak between 2 and $3 \mathrm{kHz}$, this increased intensity level in the upper formants has been related to an acoustically-perceived better voice quality with enhanced projection ${ }^{24}$ and has anatomically been related to a lowering of the larynx, thus lengthening the vocal tract, and to an increase of the ratio between the volume of the pharynx and the amplitude of the epilarynx, which is the area just above the vocal folds. ${ }^{24,25}$ The presence of an actor's formant in female voices has been postulated by Leino ${ }^{28}$ but has not been confirmed in a following study ${ }^{29}$ according to which the vocal projection of the actress' voice seemed to be acoustically represented by a lower and stronger F0 than F1 and thus resulted by glottal rather than vocal tract adjustments.

The aim of the present study is to determine the acoustical, auditory and self-perceived short-term effects of a vocal warm-up based on a protocol of SOVTE (including both fluctuating and steady exercises with decreasing resistance) in a population of actors with healthy voices. Special attention is paid to a possible effect of eliciting / enhancing the "actor's formant" in male and female voices. Moreover, pre and post show voice samples are compared.

\section{MATERIALS AND METHODS}

\section{Subjects}

Twenty-seven Italian professional theater actors ( 16 females and 11 males) with a mean age of 38.3 years (range 2272 years old) gave their informed consent to participate in this study.

Inclusion criteria were: at least 3 years of experience working and being on stage with a play during the data collection period. Exclusion criteria were: history of voice complaints which required an Ear-Nose-Throat or SpeechLanguage-Therapy consultation in the last 12 months, presence of voice complaints during the data collection period, difficulties in performing the exercises. Table 1 displays the participants' years of experience on stage.

The participants performed in five different plays with different roles, but with a comparable vocal load. The performance length varied from one hour and a half to two hours with a mean length of one hour and forty minutes.

\section{Voice recordings and SOVTE protocol}

Four audio-recordings at different times (R1, R2, R3, R4, see Table 2) were obtained for each actor while interpreting and reading aloud with acting voice a selected short passage of "Hamlet" as translated in Italian (Figure 1) and imagining an
TABLE 1.

Number and Percent of Actors with Given Years of Experience on Stage

\begin{tabular}{lcc}
\hline Years of Experience & Number of Participants & Percent \\
\hline $3-5$ & 5 & $18.52 \%$ \\
$6-10$ & 6 & $22.22 \%$ \\
$>10$ & 16 & $59.26 \%$ \\
\hline
\end{tabular}

TABLE 2.

Timeline of the Data Collection Procedures

\begin{tabular}{|c|c|c|c|c|c|}
\hline \multirow{2}{*}{$\begin{array}{l}\text { Day } 1 \\
\text { R1 }\end{array}$} & \multicolumn{5}{|c|}{ Day 2} \\
\hline & $\mathrm{R} 2$ & $\begin{array}{l}\text { SOVTE } \\
\text { Protocol }\end{array}$ & R3 & $\begin{array}{l}\text { Stage } \\
\text { Performance }\end{array}$ & $\mathrm{R} 4$ \\
\hline base & & & & & \\
\hline
\end{tabular}

audience of 400 people. The sentence "Ma che è lui per Ecuba e Ecuba per lui" was selected and extracted from the recording and considered for acoustical and auditory perceptual evaluation in order to obtain an accurate analysis. Indeed, this sentence is quite well suited for acoustical analysis, being largely vocalic. The actors were not aware of this selection.

The audio recording $\mathrm{R} 1$ was obtained the day before the show. The audio R2 was recorded on the day of the show, just before the SOVTE session, and, together with R1, made up the baseline. The SOVTE session was performed approximately one hour before the show.

The exercise session consisted of a series of 10 minutes of SOVTE progressing from high to low resistance as recommended. ${ }^{19}$ The protocol included: (1) Lax Vox with tube immersion of $3 \mathrm{~cm}$ in water according to Guzman et al, ${ }^{30} 20$ vocalizations on $/ \mathrm{u} /$; (2) straw phonation, 20 sustained $/ \mathrm{u} /$; (3) 10 lip trills, (4) 10 tongue trills, and (5) 10 hummings. The tube was $35 \mathrm{~cm}$ in length and 1 in diameter, while the straw was $10 \mathrm{~cm}$ and $3 \mathrm{~mm}$, respectively. Standard sizes of tube and straw were chosen according to Andrade. ${ }^{31}$ Participants were asked not to perform any additional warm up.

The recording R3 was obtained immediately after the exercise session, soon before the actors' performance in a show. R4 was gathered soon after the show.

Non è mostruoso che quell'attore là, fingendo
sulla scena una passione solo immaginata,
tanto assoggetti al suo concetto l'animo, da
tremar tutto, e farsi pallido smorto, gli occhi
gonfi di pianto, la voce rotta e ogni atto e gesto
protesi all'intento?
E tutto questo, per chi?
Per Ecuba.
Ma che è lui per Ecuba e Ecuba per lui, che egli
ne debba piangere? Che farebbe allora, quando
avesse alla vendetta i motivi e la spinta che ho
io?

FIGURE 1. Passage recorded at each time point (from Shakespeare, Hamlet 2.2, Italian translation by Vico Lodovici). 
The recording tool consisted of a SM58 dynamic microphone (Shure, Niles, Illinois) coupled with an US-322/366 external sound card (Tascam, Santa Fe Springs, California) and a VivoBook A551LB laptop (Asus, Taipei, Taiwan), the software Audacity (Audacity Team, 2017) set at a $44.1 \mathrm{kHz}$ sampling rate and 16 bit resolution. In order to avoid systematic differences related to the recording environment, a constant mouth-to-microphone distance of $15 \mathrm{~cm}$ was kept during each recording with the use of a metal spacer.

\section{Perceptual evaluation}

The audio recordings $\mathrm{R} 1, \mathrm{R} 2, \mathrm{R} 3$ and $\mathrm{R} 4$ were randomized for each actor and evaluated perceptually for overall voice quality by 5 blinded clinicians ( 3 voice therapists and 2 phoniatricians) who are experts in voice disorders and voice evaluation, with high quality speakers and within silent rooms. Recordings were rated using a $100 \mathrm{~mm}$ visual analogue scale whose extremes were labeled with "worst" and "best." Raters were able to play the recordings back and compare the four ones for each actor; no prior training was given.

\section{Acoustic analysis}

All the recordings were acoustically analyzed. Specifically, the following parameters were computed with the software BioVoice $^{32}$ : mean, standard deviation, lowest and highest value for fundamental frequency (F0) and for the first five formants F1-F5, jitter\%, duration (seconds), \% voiced and $\%$ unvoiced, LTAS and Quality Ratio (QR). QR is the ratio between the acoustic energy in the cluster of $F 1$ and $F 2$ and that in the cluster of F3, F4, and F5. The more QR is close to 0 , the more the speaker's formant is prominent. ${ }^{32}$ Shimmer $\%$, NHR and dynamic range $(\mathrm{dB})$, were computed with the software Praat. ${ }^{33}$ We included in our acoustic analysis not only basic voice quality parameters like jitter, shimmer and NHR, but also prosodic (F0 range, speech rate) and dynamic ( $\mathrm{dB}$ range) criteria, which were supposed to reflect the expressivity of the voice, and a measure related to the strength of the actor's formant.

\section{Perceptual self-assessment}

All participants were asked to fill in a questionnaire indicating, for the parameters: - comfort, sonorousness, expressivity, pleasantness, clarity, and power - if they had the feeling that their voice was worse, the same or better after the vocal warm-up as compared to before. Data was anonymously collected after the show.

\section{Statistical analysis}

Males and females data were analyzed both separately and as a group with SPSS version 25, the significance level was set to 0.05 . The auditory-perceptual evaluations of the 5 raters were averaged as well as all the data of R1 and R2, which made the baseline.

Inter-rater reliability was assessed through the Pearson's Correlation test. ANOVA, paired Student's $t$ test and Cohen's d effect size were used to statistically and clinically analyze pre-post session changes in the perceptual and acoustic outcomes as well as the differences between males and females. Binomial distribution and Fisher's exact test were applied to analyze the self-assessment ratings. Bonferroni correction was applied to statistically significant results.

\section{RESULTS}

All subjects correctly performed the exercises. For technical reasons, the acoustic analysis of 5 recordings failed for the parameters F0, F1 and QR, thus, the corresponding subjects (3 males, 2 females) were excluded from those parameters' analysis.

\section{Perceptual evaluation}

A weak to moderate inter-rater reliability of the listeners' evaluations was found according to Cohen's guidelines ${ }^{34}$ (Table 3).

The data analysis showed no statistically significant differences on the perceptual ratings between pre/post exercise

\section{TABLE 3.}

Pearson's $r$ and $P$ Values of Each Evaluator's Ratings Across the Others.

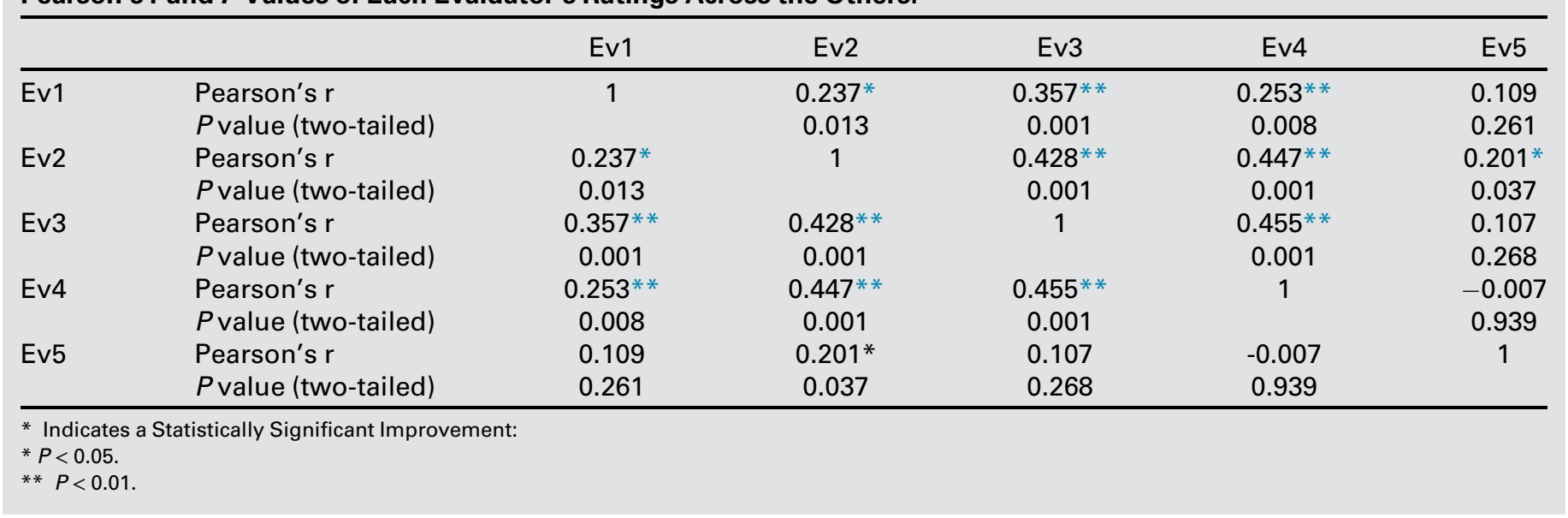




\section{TABLE 4.}

Mean \pm Standard Deviation of Actors' Perceptual Ratings at the Three Different Time Points

\begin{tabular}{lccc}
\hline & Baseline & R3 & R4 \\
\hline Males & $61.62 \pm 8.39$ & $63.96 \pm 10.69$ & $63.26 \pm 14.22$ \\
Females & $60.87 \pm 5.5$ & $62.87 \pm 16.45$ & $67.29 \pm 11.53$ \\
\hline
\end{tabular}

The ratings were measured with a value from 0 , the worst, to 100 , the best. $\mathrm{R} 3$, recording $3 ; \mathrm{R} 4$, recording 4 .

sessions and pre/post show, neither for the total group, nor for males and females separately.

Descriptive statistics for the perceptual ratings are displayed in Table 4 (averaged) and Figures 2 and 3 (males and females respectively, for each rater).

\section{Acoustic analysis}

A mild peak in the F3-F5 area of the LTAS was found, on average, for both male and female actors (Figure 4). On average, a slight increase in energy in the actor's formant range was visible in R3, following the SOVTE protocol; however, a detailed analysis of acoustic energy slice by slice (each of $500 \mathrm{~Hz}$ ) (Figure 5) showed no significant difference across the four recording times. Furthermore, QR data showed no statistically significant difference between pre and post exercise session neither for the total group, nor for males and females separately (Table 5).

No statistically significant correlation was found between the QR data and the auditory-perceptual voice ratings $(\mathrm{r}=-0.101, P=0.439$ for the female group and $\mathrm{r}=0.073$, $P=0.647$ for the male group).

The data analysis showed no statistically significant differences for the other considered acoustic parameters between pre/post exercise sessions and pre/post show, neither for the total group, nor for males and females separately. Descriptive statistics is reported in Table 5.

\section{Perceptual self-assessment}

In the total group, four out of the six parameters showed a significant improvement (ie, significantly more "better" ratings than "no change" and "worse" ratings cumulated): comfort, sonorousness, clarity and power (Figure 6).

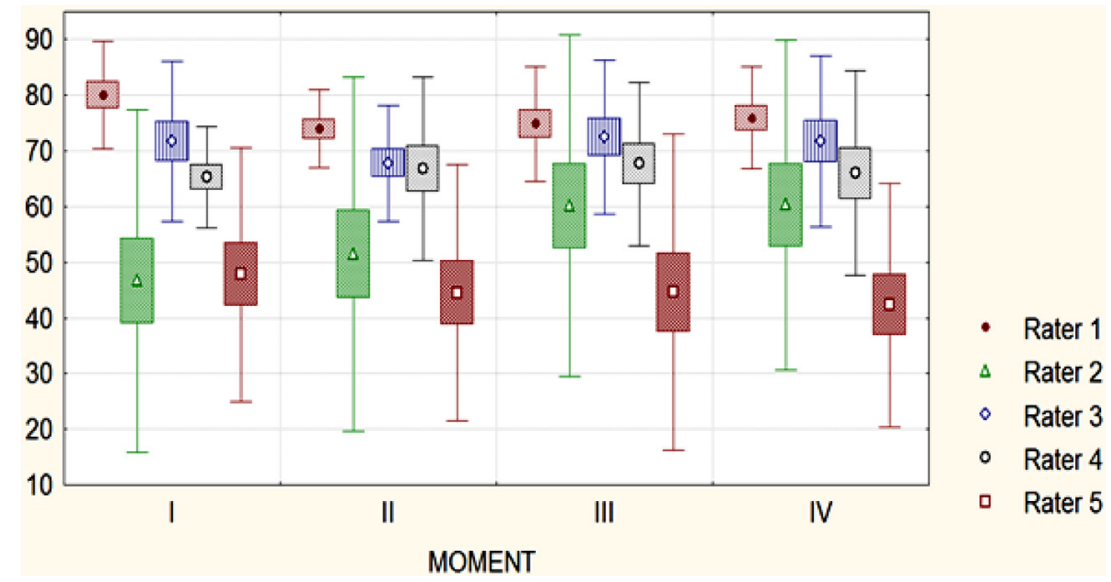

FIGURE 2. Mean, standard error (SE) and standard deviation (SD) of male actors' perceptual evaluation at each time point and for each rater.

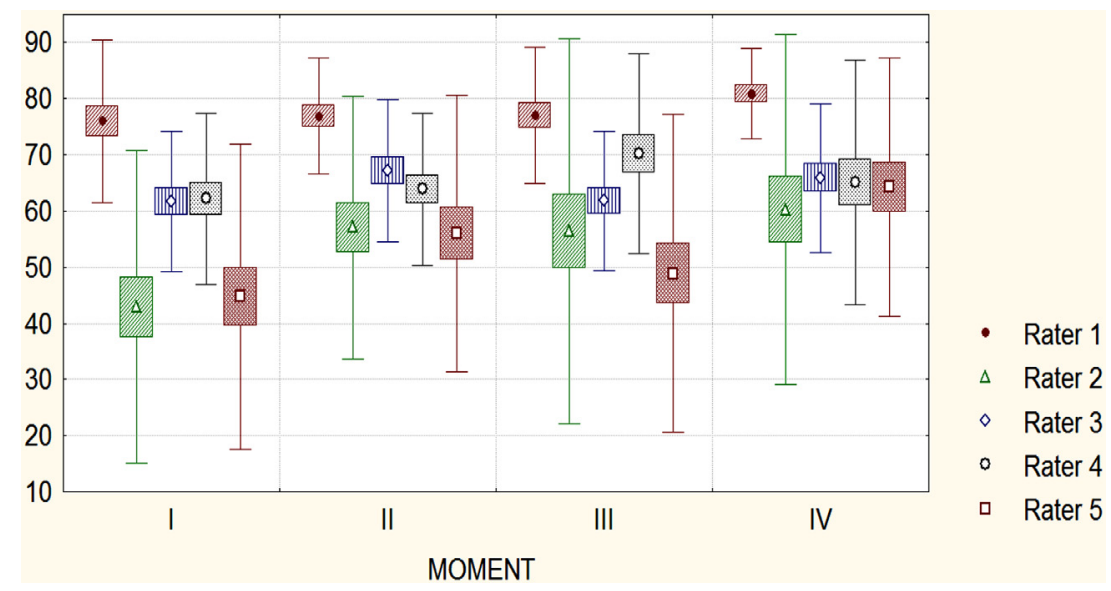

FIGURE 3. Mean, standard error (SE) and standard deviation (SD) of the actresses' perceptual evaluation at each time point and for each rater. 


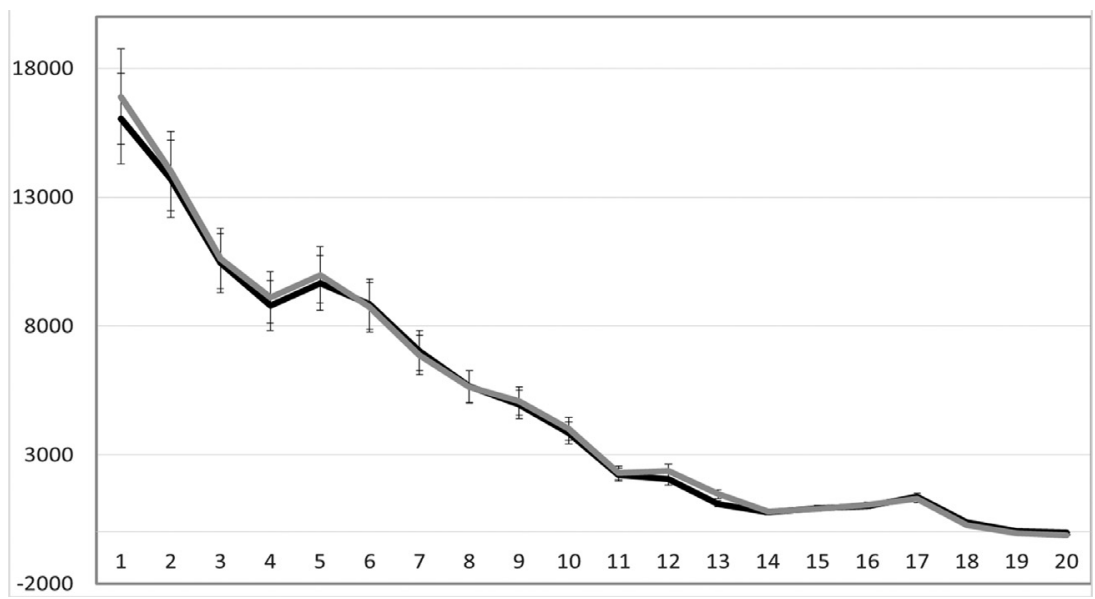

FIGURE 4. Average and standard deviation $0-10 \mathrm{kHz}$ LTAS of actors (black line) and actresses (grey line) in recording 4 . Each number on the $\mathrm{x}$-axis is relative to a $500 \mathrm{~Hz}$ frequency step.

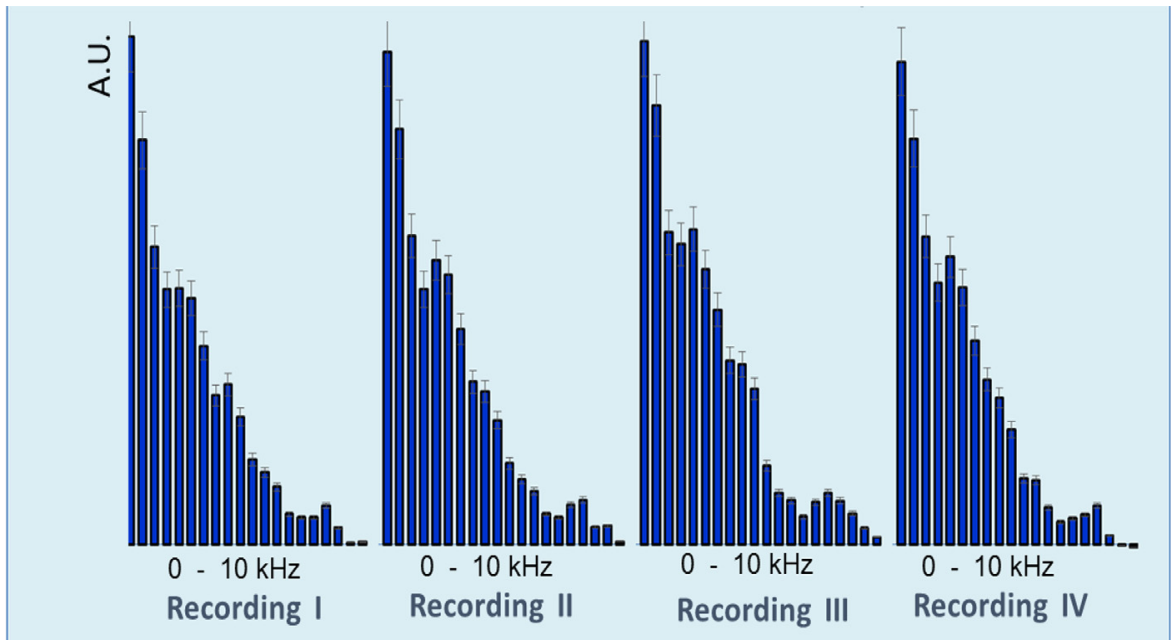

FIGURE 5. Cumulative Power Spectral Density (males and females) of the four recordings in the range $0-10 \mathrm{kHz}$. Each stripe represents mean and standard deviation energy level of a $500 \mathrm{~Hz}$ slice. A.U., Arbitrary Units.

When genders were considered separately, a statistically significant improvement $(P<0.05)$ was found in the parameters concerning comfort in production for males, sonorousness and vocal clarity for both males and females, power for females. The percentage of actors who noticed an improvement is shown in Table 6.

\section{DISCUSSION}

The present study aimed to define the short-term effects of a SOVTE warm-up protocol on the actor's voice.

After 10-minutes of vocal warm-up, no statistically significant differences were found across the four recording times on either the acoustic or the auditory-perceived voice quality parameters. These findings differ from the ones obtained in the previous work of Leino et $\mathrm{al}^{24}$ just on one single actor with a 30-minute vocal warm-up. Our SOVTE protocol, however, was short enough to be easily applied before the stage performance and to avoid a fatigue effect on the voice before the performance. To the best of our knowledge, the present study is the first one carried out in a real environment and during the stage performance period rather than in an artificial context such as a voice laboratory. This type of real-life setting may have influenced the quality of the recordings, which were made in theaters, as well as the physical and emotional condition of the actors. This might be considered a limitation of the study, nevertheless the conditions in which the present data were collected are the typical ones of the real working context of the actors.

The findings presented above are in line with the previous literature about the effects of SOVTE on healthy voices. Costa et $\mathrm{al}^{35}$ did not find any significant differences in the auditory perceptual and acoustic assessments in this population. Laukkanen et al found a significant increase in F3 and decrease in F4 in women without other significant changes, concluding that exercising with SOVTE may help in determining a speaker's formant cluster.

It is known that actresses show slightly more acoustical energy in the $3-4 \mathrm{kHz}$ zone of the long-time averaged 
TABLE 5.

Mean \pm Standard Deviation of Actor's Acoustic Parameters at the Three Different Time Points

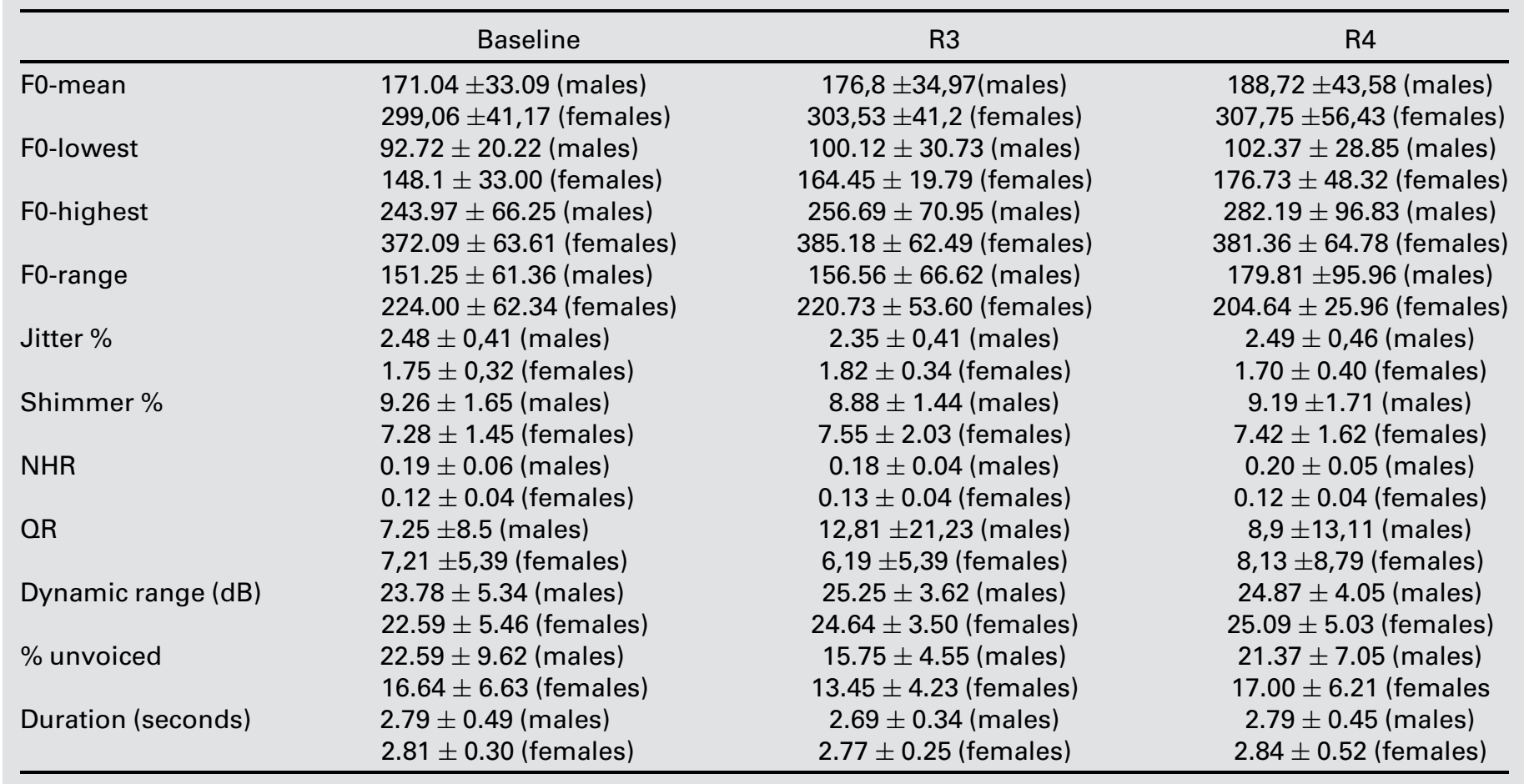

F0, fundamental frequency; NHR, noise to harmonic ratio; QR, quality ratio; R3, recording 3; R4, recording 4.

spectrum in the acting condition, compared to the nonacting condition, although this difference does not look like a true "formant," as observed in male singers, and even in male actors. ${ }^{36}$ However, in our experiment, all recordings were in "acting" condition. Our findings do not suggest that the SOVTE-exercises elicit or reinforce an actor's formant.

The interrater correlation for the auditory perceptual evaluation appears to be quite low, notwithstanding the experience of the raters. However, it must be considered that the present experiment only deals with non-pathological voices, while all raters are clinicians, not vocal artists.
Moreover, the findings of this study showed that a 10minute vocal warm-up with SOVTE is enough to determine a self-perceived improvement in the comfort of production for males and in sonorousness, voice clarity and power for both male and female actors. This result is in line with the previous literature on the effects of SOVTE on the singer's voice. ${ }^{17}$ A questionnaire similar to the one described in our study was used by Fantini et al for healthy singers. ${ }^{22}$ To the best of our knowledge, no tools with a focus on self-assessment parameters for healthy vocal performers have been validated. It is to be recommended that future research in this field should deal with creating reliable/valid tools for

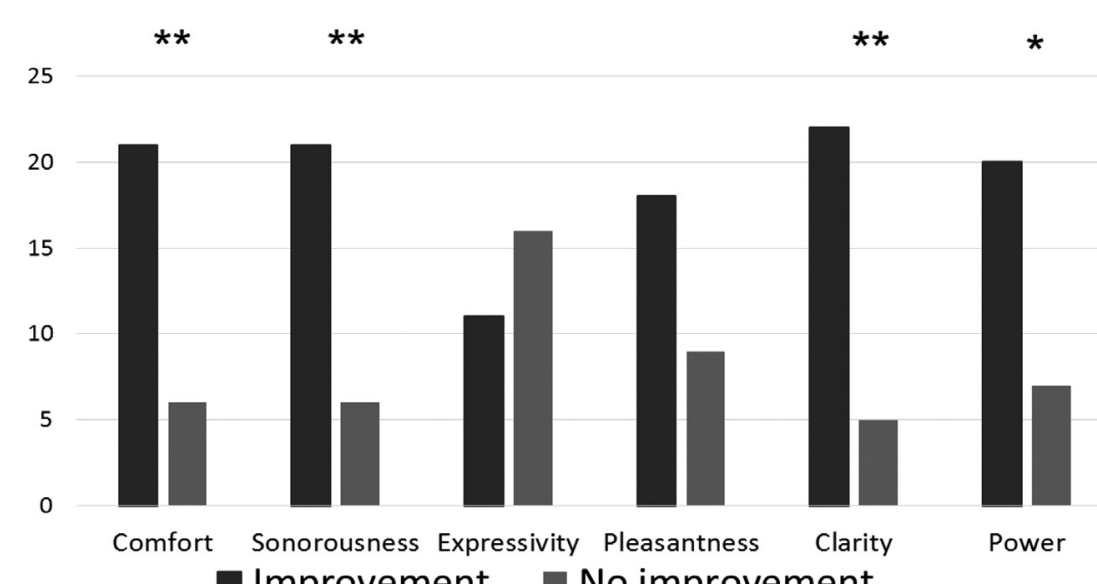

FIGURE 6. Male and female actors' responses for each self-assessment parameter. * indicates a statistically significant improvement: *0.01 $<P<0.02, * * 0.001<P<0.01$. 
TABLE 6.

Percentage of Male and Female Actors who Found an Improvement on Each Self-Assessment Parameter

\begin{tabular}{|c|c|c|c|c|c|c|}
\hline & Comfort & Sonorousness & Expressivity & Pleasantness & Clarity & Power \\
\hline Females & $54.54 \%$ & $81.81 \% *$ & $45.45 \%$ & $72.72 \%$ & $72.72 \% *$ & $81.81 \%$ * \\
\hline
\end{tabular}

* Indicates a Statistically Significant Improvement: ${ }^{*} 0.04<P<0.03$.

** $0.02<P<0.01$.

$* * * 0.001<P<0.01$

measuring the self-perception of voice quality in artists, with sufficient sensitivity to detect subtle changes in the normophonic voice.

Given that the voice function is multidimensional, several different outcome measures have been used to investigate the effect of SOVTE in previous studies. These include both objective measures as electroglottography, computerized tomography, acoustics, quantification of the intraoral and subglottic pressure, aerodynamic and subjective measures as self-assessment and the auditory-perceptive analysis. ${ }^{15-18}$ Future research on the effects of SOVTE on the actor's voice could employ other instrumental analyses.

In this study, auditory-perceived voice quality and acoustic parameters did not significantly change after the performance. This result is in line with the previous literature. ${ }^{37} \mathrm{~A}$ single performance of about one and a half hour, indeed, seems not able to affect the objective vocal quality as measured soon after the show. ${ }^{37}$ Future research might clarify if SOVTE are able to prevent the negative impact of a longer period of stage performance.

Different kinds of vocal warm-up exercises might be included in future studies in order to better clarify the impact of SOVTE on self-perceived voice quality.

In conclusion, this study demonstrated that for the actors a short vocal warm-up based on SOVTE may be effective in determining a self-perceived improvement in comfort of production, voice quality and strength. Although we achieved an exhaustive acoustic analysis, the objective assessment methods might not demonstrate changes which are only self-perceivable. The primary goal of voice clinicians is to help clients in developing a voice which is selfperceived as more comfortable, easier and stronger; therefore, SOVTE could be an easy means to warm up the voice before the show and the rehearsals for performing actors. Further, there are few doubts that self-confidence is important in performing artists.

\section{Acknowledgments}

The authors are grateful to Annamaria Zambarbieri and Cristiana Assi for participating in the perceptual ratings of the recordings, and thankful to each actor who adhered to the study.

\section{SUPPLEMENTARY DATA}

Supplementary data related to this article can be found online at doi:10.1016/j.jvoice.2020.07.024.

\section{REFERENCES}

1. Roy N, Ryker KS, Bless DM. Vocal violence in actors: an investigation into its acoustic consequences and the effects of hygienic laryngeal release training. $J$ Voice. 2000;14:215-230.

2. Emerich KA, Titze IR, Švec JG, et al. Vocal range and intensity in actors: a studio versus stage comparison. J Voice. 2005;19:78-83.

3. Kitch JA, Oates J. The perceptual features of vocal fatigue as selfreported by a group of actors and singers. J Voice. 1994;8:207-214.

4. Lerner MZ, Paskhover B, Acton L, et al. Voice disorders in actors. $J$ Voice. 2013;27:705-708.

5. Rangarathnam B, Paramby T, McCullough GH. "Prologues to a bad voice": effect of vocal hygiene knowledge and training on voice quality following stage performance. J Voice. 2018;32:300-306.

6. Timmermans B, De Bodt MS, Wuyts FL, et al. Poor voice quality in future elite vocal performers and professional voice users. $J$ Voice. 2002;16:372-382.

7. Ribeiro VV, Frigo LF, Bastilha GR, et al. Vocal warm-up and cooldown: systematic review. Rev CEFAC. 2016;18:1456-1465.

8. Mendonça R, Sampaio T, Oliveira D. Assessing of a vocal functional exercise program of stemple and gerdeman applied to teachers. Rev CEFAC. 2009; 12:471-482.

9. Van Lierde KM, D'haeseleer E, Baudonck N, et al. The impact of vocal warm-up exercises on the objective vocal quality in female students training to be speech language pathologists. J Voice. 2011;25: e115-e121

10. McHenry M, Johnson J, Foshea B. The effect of specific versus combined warm-up strategies on the voice. J Voice. 2009;23:572-576.

11. Moreira T, Cassol M, Fávero S, et al. Vocal intervention for telemarketing service consultants: vocal well-being. Rev CEFAC. 2010;12:936-944.

12. Silverio K, Gonçalves CGO, Penteado R, et al. Actions in vocal health: a proposal for improving the vocal profile of teachers. PróFono Rev Atualização Científica. 2008;20:177-182.

13. Masson MLV, Loiola CM, Fabron EMG, et al. Aquecimento e desaquecimento vocal em estudantes de pedagogia. Distùrbios Comun. 2013;25:177-185

14. Pereira LP, Masson MLV, Carvalho FM. Vocal warm-up and breathing training for teachers: randomized clinical trial. Rev Saùde Pùblica. 2015;49:1-8.

15. Kapsner-Smith MR, Hunter EJ, Kirkham K, et al. A randomized controlled trial of two semi-occluded vocal tract voice therapy protocols. J Speech, Lang Hear Res. 2015;58:535-549.

16. Guzman M, Jara R, Olavarria C, et al. Efficacy of water resistance therapy in subjects diagnosed with behavioral dysphonia: a randomized controlled trial. J Voice. 2017;31. 385.e1-385.e10.

17. Mendes ALF, Dornelas do Carmo R, Dias de Araújo AMG, et al. The effects of phonation into glass, plastic, and LaxVox tubes in singers: a systematic review. J Voice. 2019;33. 381.e1-381.e9.

18. Guzman M, Laukkanen A-M, Krupa P, et al. Vocal tract and glottal function during and after vocal exercising with resonance tube and straw. J Voice. 2013;27. 523.e19-523.e34.

19. Titze IR. Voice training and therapy with a semi-occluded vocal tract: rationale and scientific underpinnings. $J$ Speech, Lang Hear Res. 2006;49:448-459.

20. Guzman M, Miranda G, Olavarria C, et al. Computerized tomography measures during and after artificial lengthening of the vocal tract in subjects with voice disorders. J Voice. 2017;31:124.e1-124.e10. 
21. Amarante Andrade P, Wood G, Ratcliffe P, et al. Electroglottographic study of seven semi-occluded exercises: LaxVox, straw, lip-trill, tongue-trill, humming, hand-over-mouth, and tongue-trill combined with hand-over-mouth. $J$ Voice. 2014;28:589-595.

22. Fantini M, Succo G, Crosetti E, et al. Voice quality after a semioccluded vocal tract exercise with a ventilation mask in contemporary commercial singers: acoustic analysis and self-assessments. $J$ Voice. 2017;31:336-341.

23. Guzman M, Angulo M, Munoz D, et al.. Effect on long-term average spectrum of pop singers' vocal warm-up with vocal function exercises. Int J Speech Lang Pathol. 2013:127-135.

24. Leino T, Laukkanen A-M, Radolf V. Formation of the actor's/speaker's formant: a study applying spectrum analysis and computer modeling. $J$ Voice. 2011;25:150-158.

25. Bele IV. The speaker's formant. J Voice. 2006;20:555-578.

26. Master S, De Biase N, Chiari BM, et al. Acoustic and perceptual analyses of Brazilian male actors' and Nonactors' voices: long-term average spectrum and the 'Actor's formant'. J Voice. 2008;22:146-154.

27. Pinczower R, Oates J. Vocal projection in actors: the long-term average spectral features that distinguish comfortable acting voice from voicing with maximal projection in male actors. $J$ Voice. 2005;19:440 453.

28. Leino T. Voice Quality of Finnish Female Actors. 2001. Stockholm, Sweden.
29. Master S, De Biase NG, Madureira S. What about the 'actor's formant' in actresses' voices? J Voice. 2012;26:e117-e122.

30. Guzman M, Castro C, Madrid S, et al. Air pressure and contact quotient measures during different semioccluded postures in subjects with different voice conditions. $J$ Voice. 2016;30. 759.e1-759.e10.

31. Amarante Andrade P, Wistbacka G, Larsson H, et al. The flow and pressure relationships in different tubes commonly used for semioccluded vocal tract exercises. $J$ Voice. 2016;30:36-41.

32. Manfredi C, Barbagallo D, Baracca G, et al. Automatic assessment of acoustic parameters of the singing voice: application to professional western operatic and Jazz singers. $J$ Voice. 2015;29:517.e1-517.e9.

33. Boersma P, Weenik D. Praat: doing phonetics by computer [computer program]. 2018.

34. Cohen J. Statistical Power Analysis for the Behavioral sciences - Second Edition. Lawrence Erlbaum Associates; 1988.

35. Costa CB, Costa LHC, Oliveira G, et al. Immediate effects of the phonation into a straw exercise. Braz J Otorhinolaryngol. 2011;77:461465 .

36. Dejonckere PH, Stoffels HE. Spectral specificities of acting voice in professional actresses. In: Manfredi C, ed. Proceedings of the Models and Analysis of Vocal Emissions for Biomedical Applications 11th International Workshop. Ed. Florence University Press; 2019:163-166.

37. D'haeseleer E, Meerschman I, Claeys S, et al. Vocal quality in theater actors. $J$ Voice. 2017;31:510.e7-510.e14. 